\title{
A 10-Year Follow-Up of Two-Incision and Modified Watson-Jones Total Hip Arthroplasty in Patients with Osteonecrosis of the Femoral Head
}

\author{
Shih-Jie Lin, ${ }^{1}$ Tsan-Wen Huang, ${ }^{1,2}$ Po-Chun Lin, ${ }^{3}$ Feng-Chih Kuo, ${ }^{3}$ Kuo-Ti Peng, ${ }^{1,2}$ \\ Kuo-Chin Huang, ${ }^{2,3}$ and Mel S. Lee ${ }^{2,3}$ \\ ${ }^{1}$ Department of Orthopaedic Surgery, Chang Gung Memorial Hospital, Chiayi, Taiwan \\ ${ }^{2}$ Change Gung University, Taoyuan, Taiwan \\ ${ }^{3}$ Department of Orthopaedic Surgery, Kaohsiung Chang Gung Memorial Hospital, Kaohsiung, Taiwan
}

Correspondence should be addressed to Tsan-Wen Huang; b8601081@adm.cgmh.org.tw and Mel S. Lee; bone@doctor.com

Received 25 January 2017; Revised 24 February 2017; Accepted 1 March 2017; Published 12 March 2017

Academic Editor: Akihiro Sudo

Copyright (C) 2017 Shih-Jie Lin et al. This is an open access article distributed under the Creative Commons Attribution License, which permits unrestricted use, distribution, and reproduction in any medium, provided the original work is properly cited.

\begin{abstract}
Long-term data and information indicating whether minimally invasive surgery (MIS) approaches are safe and effective with total hip arthroplasty (THA) are lacking. Between 2004 and 2006, 75 patients with alcohol-related osteonecrosis of the femoral head $(\mathrm{ONFH})$ who underwent 75 THAs with the two-incision approach were studied. The medical records, radiographic parameters, and functional outcomes were collected prospectively. All data were compared with those for matched patients who underwent a modified Watson-Jones (WJ) approach. THA using the two-incision approach was associated with longer operation time, more blood loss, more lateral femoral cutaneous nerve injury, and more periprosthetic femoral fractures $(p<0.05$ for all four) than the modified WJ approach. The Harris Hip Score (HHS) and Western Ontario and McMaster University Osteoarthritis Index (WOMAC) increased significantly from the period preoperatively to 6 weeks postoperatively and thereafter up to the last followup in both groups. However, there were no significant differences in terms of radiographic parameters and functional outcomes between the two groups throughout the study period. Both the two-incision and the modified WJ approach provided satisfactory results and survival rates at a mean follow-up of 10.8 years. A prospective, randomized, large-scale cohort study is still warranted for evidence-based recommendations.
\end{abstract}

\section{Introduction}

Total hip arthroplasty (THA) is an effective surgery for patients with end-stage osteonecrosis of the femoral head $(\mathrm{ONFH})[1]$. However, the surgical approach is one of the main confounding factors for the outcome [2,3]. Abnormal hip loading patterns have been noted after conventional THA and may affect longevity due to high cycle numbers [4]. Minimally invasive surgery (MIS) approaches to THA have been introduced in response to increased patient demands and expectations [2-8]. Early reports suggest that MIS lessens blood loss, pain, and hospital stay and results in early functional recovery compared with conventional approaches [57]. However, the merits of different MIS approaches are still controversial, and functional outcomes among the different surgical approaches vary $[4,8]$. In general, MIS approaches can be divided into those using an abridged incision, such as transgluteal or posterolateral approaches, and those using a muscle-sparing approach, such as the two-incision and modified Watson-Jones (WJ) approaches [6-8]. From the technical perspective, the muscle-sparing approach provides adequate tissue tension and stability for THAs [9-11]. Better joint stability prevents microseparation or subluxation during gait cycles and avoids edge-loading-related accelerated polyethylene wear and sequential periprosthetic osteolysis and mechanical loosening $[12,13]$.

Concerns exist regarding the safety, efficacy, and longevity of MIS THA [4-8]. The available information from clinical reports is inadequate to suggest that surgeons should change from their standard approach [3]. Comparative studies of minimally invasive direct-anterior, minimally invasive direct-lateral, minimally invasive posterior, minimally 
invasive posterolateral, minimally invasive anterolateral, and two-incision approaches have been reported [14-18]. The inconsistency of the results may be partially due to the different surgical approaches (abridged incision or musclesparing approach) used with the study populations. While a few comparisons of the two-incision technique and the modified WJ technique have been reported, the literature on long-term outcome is sparse $[4,8]$. The purpose of this study was to analyze the clinical outcomes and 10-year follow-up of ONFH patients who underwent THAs using the minimally invasive two-incision and modified WJ approaches.

\section{Materials and Methods}

This retrospective study was approved by the Ethics Committee and Institutional Review Board of our institution, and all patients provided signed informed consent.

The records of all patients who had undergone THA at our institution since 2004 were routinely entered into our database. After a clear explanation of the merits and disadvantages of different surgical approaches, the patients could choose the type of approach they wanted. We prospectively collected clinical data on age, gender, diagnosis, length of hospital stay, surgical approach used, total blood loss, and complications, as well as preoperative and postoperative radiographic and clinical functional assessments for each patient.

From 2004 to 2006, 262 patients with alcohol-related ONFH underwent a total of 316 metal-on-polyethylene THAs performed by a single surgeon. We manually identified patients who had unilateral hip involvement and chose to undergo a two-incision approach. The Fiber Metal Taper stem (Versys, Zimmer), used with a cementless press-fitting technique, and a highly cross-linked polyethylene-bearing surface were used in all THAs. Those patients with (a) previous surgeries on the hip joint, (b) a preexisting hip deformity, (c) bilateral THAs, and (d) incomplete medical records, radiographic analyses, or clinical functional assessments were excluded.

Seventy-five patients met our criteria. The study group consisted of 65 men and 10 women with a mean age of 44 years (range: 30 to 60 years) at the time of surgery. Age, gender, and date-of-surgery-matched patients with $\mathrm{ONFH}$ who had undergone THAs using the same prostheses and a modified WJ approach performed by the same surgeon were used as controls. To determine adequate sample size, an $a$ priori power analysis using the hypothesis test with a power of $90 \%$ and a significance of 0.05 was performed. According to Achten et al. [19], 48 hips were required per group to detect a difference of 10 points in the Harris Hip Score (HHS) (estimated standard deviation of 15). A cut-off value was selected because a difference of 10 points was suggested as the minimal clinically important difference.

All patients enrolled in this study were treated with the same protocol. On the basis of the standard of care following cementless THA, the patients were encouraged to ambulate with partial weight-bearing as soon as possible after surgery, under the supervision of a physical therapist. All patients used crutches or a walker with full weight-bearing for 6 weeks and a cane when needed thereafter.
Radiological assessments included the cup inclination, the cup anteversion, the presence of radiolucent lines in the prosthesis-bone interface, the alignment and canal fill ratio of the femoral stem, and the limb-length discrepancy, as previously described [20-22]. The radiographic assessments were reviewed and analyzed by an independent surgeon who was blinded to the groupings and patient demographic data. Intraobserver reliability was assessed according to the method described by Konigsberg et al. [23] and was rated as good to very good. Clinical results were assessed using the HHS [24] and Western Ontario and McMaster University Osteoarthritis Index (WOMAC) [25], beginning preoperatively and at intervals of 6 weeks, 3 months, and 6 months and yearly after the surgery. All functional outcomes were assessed by an independent observer who was also blinded to the groupings and patient demographic data.

Complications were recorded. Any medical or surgical event that compromised the clinical recovery of the patients, such as wound infection, venous thromboembolism (VTE), neurovascular injury, fracture, dislocation, implant malposition, implant size mismatch, or early loosening, was defined as an adverse event.

2.1. Statistical Analysis. Statistical analysis was performed by an independent statistician using the SPSS for Windows statistical package (version 17.0, SPSS, Chicago, Illinois). Differences among patients who underwent the two-incision approach and control patients were examined using the $\chi^{2}$ test for categorical variables, a nonparametric test for ordinal variables, and the $t$-test for continuous variables. Significance was set at $p<0.05$.

\section{Results}

The demographic and perioperative variables of patients undergoing the two-incision approach and control patients are shown in Table 1. Patients undergoing the two-incision approach had a longer operation time (160 minutes versus 117 minutes, $p<0.001)$ and more blood loss $(719 \mathrm{~mL}$ versus $366 \mathrm{~mL}, p<0.001)$ than did patients in the WJ group. With regard to length of hospital stay and wound length, there was no significant differences between the 2 groups.

Radiological analysis revealed no significant differences in cup inclination, cup anteversion angles, stem alignment, canal fill ratio, or limb-length discrepancy. The percentages of procedures that had ideal positioning were similar between the 2 groups (Table 2).

Seven patients that had periprosthetic fracture (6 in the two-incision group and 1 in the WJ group) were excluded from the final analysis because there would be different rehabilitation protocols and influences on outcome assessment. The remaining hips were included in the final functional analysis. There were no significant differences in HHS and WOMAC scores in either group before surgery. The HHS and WOMAC scores increased significantly from baseline to 6 weeks and thereafter up to the most recent follow-up in both groups $(p<0.001)$. The 2 groups did not differ in functional outcomes during the entire study period (Table 3 ). 
TABLE 1: Demographic data on the two-incision and Watson-Jones groups.

\begin{tabular}{lccc}
\hline Demographic information & Two-incision group & WJ group & $p$ value \\
\hline Age (years) & $44 \pm 8$ & $44 \pm 8$ & Matched \\
Gender (male : female) & $65: 10$ & $65: 10$ & Matched \\
Side of operation (number of hips) & & $41(54.7 \%)$ & 0.988 \\
$\quad$ Right & $42(56.0 \%)$ & $34(45.3 \%)$ & - \\
$\quad$ Left & $33(44.0 \%)$ & $117 \pm 36$ & - \\
Operation time (min) & $160 \pm 41$ & $366 \pm 208$ & $<0.001^{*}$ \\
Perioperative blood loss (mL) & $719 \pm 423$ & $5 \pm 2$ & $0.001^{*}$ \\
Length of hospital stay (day) & $5 \pm 1$ & $9 \pm 2$ & 0.677 \\
Wound length (cm) & $10 \pm 1$ & & \\
\hline
\end{tabular}

WJ = Watson-Jones.

Values are shown as mean (standard deviation) or as $n$ (\%).

$p$ values for between-group comparisons were determined by the chi-squared test and Fisher's exact test for categorical variables and Student's $t$-test for continuous variables.

${ }^{*}$ Statistically significant $(p<0.05)$.

TABLE 2: Comparison of radiographic results in the two-incision and Watson-Jones groups.

\begin{tabular}{lccc}
\hline & Two-incision group & WJ group & $p$ value \\
\hline Cup inclination angle $(\mathrm{deg})$ & $44 \pm 5$ & $45 \pm 4$ & 0.052 \\
Number of outliers & $4(5.3 \%)$ & $7(9.3 \%)$ & 0.325 \\
(cups with angle of $\leq 30^{\circ}$ or $\left.\geq 50^{\circ}\right)$ & $17.5 \pm 7.7$ & $15.7 \pm 6.4$ & 0.105 \\
Cup anteversion $(\mathrm{deg})$ & $17(22.7 \%)$ & $9(12.0 \%)$ & 0.099 \\
Number of outliers & & & \\
(cups with angle of $\leq 5^{\circ}$ or $\left.\geq 25^{\circ}\right)$ & Valgus & $-0.2 \pm 1.2$ & 0.127 \\
Stem alignment $(\mathrm{deg})$ & $0.1 \pm 1.1$ & $94.0 \pm 4.2$ & 0.661 \\
Canal fill ratio $(\%)$ & $94.0 \pm 4.1$ & $0.8 \pm 2$ & 0.510 \\
Limb-length discrepancy $(\mathrm{mm})$ & $0.7 \pm 1.5$ & & \\
\hline
\end{tabular}

Values are shown as mean (standard deviation) or as $n$ (\%).

$p$ values for between-group comparisons were determined by the chi-squared test and Fisher's exact test for categorical variables and Student's $t$-test for continuous variables.

TABLE 3: Functional results of the two-incision and Watson-Jones groups.

\begin{tabular}{lccc}
\hline & Two-incision group & WJ group & $p$ value \\
\hline \multicolumn{4}{c}{ Harris Hip Score } \\
\hline Preoperative & $57 \pm 12$ & $56 \pm 11$ & 0.151 \\
6 weeks & $90 \pm 6$ & $91 \pm 6$ & 0.982 \\
3 months & $94 \pm 5$ & $94 \pm 5$ & 0.961 \\
6 months & $96 \pm 4$ & $96 \pm 4$ & 0.933 \\
1 year & $98 \pm 3$ & $93 \pm 4$ & 0.868 \\
Last follow-up & $94 \pm 6$ & $56 \pm 8$ & 0.799 \\
\hline & Western Ontario and McMaster University Osteoarthritis Index & \\
\hline Preoperative & $58 \pm 9$ & $89 \pm 8$ & 0.222 \\
6 weeks & $91 \pm 5$ & $96 \pm 5$ & 0.956 \\
3 months & $95 \pm 5$ & $99 \pm 4$ & 0.971 \\
6 months & $96 \pm 4$ & $99 \pm 3$ & 0.854 \\
1 year & $99 \pm 5$ & $96 \pm 5$ & 0.822 \\
Last follow-up & $95 \pm 4$ & & 0.868 \\
\hline
\end{tabular}

$\mathrm{WJ}=$ Watson-Jones.

Values are shown as mean (standard deviation).

$p$ values for between-group comparisons were determined using Student's $t$-test. 
TABLE 4: Complications in the two-incision and Watson-Jones groups.

\begin{tabular}{lccc}
\hline & Two-incision group & WJ group & $p$ value \\
\hline Lateral femoral cutaneous nerve palsy & $20(26.7 \%)$ & 0 & $<.001^{*}$ \\
Periprosthetic femoral fracture & $6(8.0 \%)$ & $1(1.3 \%)$ & $0.049^{*}$ \\
Cup loosening & 0 & $1(1.3 \%)$ & 0.493 \\
Stem loosening & $2(2.7 \%)$ & 0 & 0.242 \\
Superficial wound infection & $3(4.0 \%)$ & $1(1.3 \%)$ & 0.118 \\
Dislocation of the hip & 0 & $3(4.0 \%)$ & 0.493 \\
Hips with complications $(n)$ & $31(41.3 \%)$ & $<0.001^{*}$ \\
\hline
\end{tabular}

Values are shown as mean (standard deviation) or as $n$ (\%).

$p$ values for between-group comparisons were determined by the chi-squared test and Fisher's exact test.

${ }^{*}$ Statistically significant $(p<0.05)$.

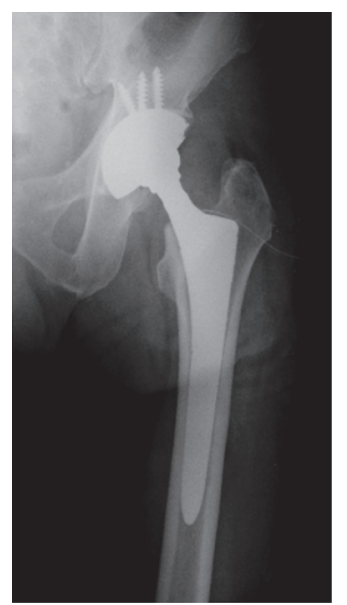

(a)

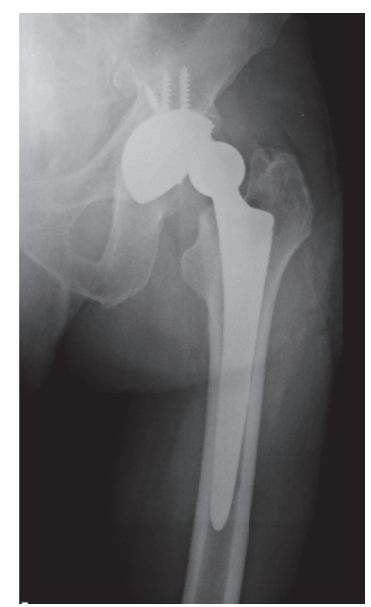

(b)

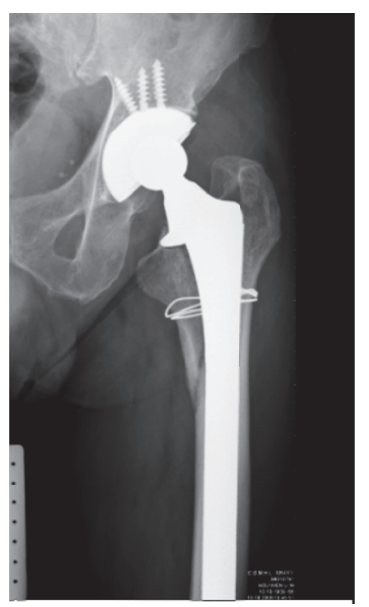

(c)

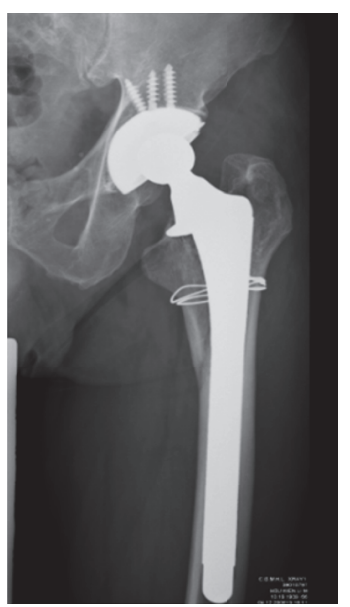

(d)

FIGURE 1: A 62-year-old male with alcohol-related ONFH underwent THA with a two-incision approach. (a) Immediate postoperative left hip anteroposterior view. (b) Loosening of femoral stem and hip dislocation occurred in the postoperative 6 months. (c) Revision of femoral component with long stem was performed. (d) Ten years later, the hip remains with good stability and adequate position.

There was no difference in complications between the 2 groups, except for lateral femoral cutaneous nerve palsy $(p<0.001)$ and periprosthetic fracture $(p=0.049)$. A summary of complications during the 10-year follow-up period revealed that no deep infection, VTE, or implant size mismatch had occurred in either group. Both groups showed similar findings with regard to cup loosening, stem loosening, superficial wound infection, and dislocation of the hip. The overall complication rate was significantly higher in the two-incision group $(41.3 \%)(p<0.001)$ (Table 4$)$.

Twenty hips in the two-incision group were complicated with lateral femoral cutaneous nerve palsy in the thigh. At the last follow-up, 3 of them still had residual symptoms. However, there were no nerve palsy complications in the WJ group, a significant difference $(p<0.001)$. One hip in the WJ group sustained an intraoperative periprosthetic fracture of the proximal femur that needed additional cerclage wire fixation. In contrast, 6 hips in the two-incision group sustained an intraoperative periprosthetic femoral fracture, 4 of which were fixed with cerclage wires during the index operation and 2 of which were detected in the postoperative stage when the femoral stem subsided. The latter 2 fractures needed a second surgical procedure to revise the femoral component.
One loose cup was found in the WJ group in the late stage. Two femoral stems in the two-incision group but none in the WJ group were loose. The loose femoral stems, both of which were revised, were diagnosed 6 and 9 months after the index operation (Figure 1). Using component revision as the endpoint for survival analysis, the survival rate for cups was $100 \%$ in the two-incision group and $98.7 \%$ in the WJ group. The survival rate for stems was $97.3 \%$ in the two-incision group and $100 \%$ in the WJ group. Three hips in the twoincision group had superficial wound infection, and all were successfully treated with antibiotics immediately; there was no wound infection in the WJ group. There was one hip dislocation, a posttrauma posterior dislocation, in the WJ group 3 months after the index operation. Closed reduction was successful, and there were no additional dislocations (Table 4).

\section{Discussion}

The most important finding in this investigation is that use of the modified WJ approach for THA in ONFH hips resulted in shorter operation time, less blood loss, and a lower incidence of lateral femoral cutaneous nerve injury and periprosthetic femoral fracture compared to the two-incision approach. 
However, there was no statistically significant difference between the 2 groups in radiographic parameters, functional outcomes, and survival rate at a mean follow-up of 10.8 years.

MIS THA has gained popularity in recent years. To date, however, no consensus exists regarding the merits, safety, and efficacy of MIS THA [4-8]. Nevertheless, questions have been raised regarding whether iatrogenic complications such as fractures or nerve injuries can be prevented and whether components can be placed in their correct position through small incisions [11]. Comparative studies of abridged incision approaches and muscle-sparing approaches have been reported, but little has been published regarding comparisons of muscle-sparing techniques [4-8]. In addition, long-term data regarding the longevity of MIS THA is lacking, as is information indicating whether MIS approaches are safe and effective with THA [5-8].

There are 2 comparative studies on two-incision THA and modified WJ THA in the literature. In 2012, we published a prospective randomized study on patients who had a twoincision THA in one hip and a modified WJ THA in the other to investigate the efficacy of the 2 techniques and found no significant difference in the radiographs and clinical outcomes of the patients [8]. However, several limitations must be acknowledged. First, the study was limited by its short-term clinical follow-up. Second, only 20 patients (40 hips) were studied. Third, the reasons for undergoing THA included both ONFH and osteoarthritis (OA). In the second study, Foucher et al. [4] conducted a randomized controlled trial involving 32 patients with $\mathrm{OA}$. The purpose of the trial was to determine the efficacy of the two-incision and modified WJ approaches, individually, during the first postoperative year. No significant differences were detected between the 2 techniques in terms of gait analysis and time-course of recovery. However, similar to our study, the evidence was limited by the small number of patients and the short-term follow-up.

In this study, the operation time was longer in the twoincision group. The surgical field could not be fully visualized during implantation of the femoral stem and repeated evaluation of its position by intraoperative fluoroscopy may have resulted in longer operation times and more blood loss in this group. Lateral femoral cutaneous nerve injury occurred only in the two-incision group, with an incidence rate of $26.7 \%$, which is comparable to the $24.7 \%$ rate in another study [26]. This recognized risk is associated with the various anterior approaches to the hip joint, including the twoincision approach $[26,27]$. To avoid this complication, some surgeons use the intermuscular interval between the gluteus medius and the tensor fascia latae (the WJ interval) in their two-incision techniques [28]. Our findings are compatible with their results: the WJ group had fewer cases with lateral femoral cutaneous nerve injury.

Periprosthetic fractures in the perioperative or early postoperative stage are primarily iatrogenic [29-31]. In this study, we had a periprosthetic femoral fracture rate of $8.0 \%$ in the two-incision group (1.3\% in the WJ group), which was higher than the $2.5 \%$ fracture rate reported in a Chinese study that used a cementless press-fit technique with ONFH hips [32]. In ONFH hips, the lesion can be extensive and involve the area below the lesser trochanter in 5\% to $11 \%$ of hips [32-34]. In addition, patients with ONFH have been reported to be "at risk" for abnormal hip anatomy with a low neck-shaft angle and high femoral neck version. These anatomical variations and the limited surgical exposure might cause reconstruction difficulties and possibly contribute to more surgeon-related complications when using minimally invasive approaches [35]. For cup implantation, the two-incision and modified WJ approaches are similar, and the only difference is in the different intermuscular interval used. For implantation of the femoral stem, however, the 2 techniques are quite different. One incision for the cup and another for the stem should theoretically be feasible for implanting a prosthesis and precluding complications like a periprosthetic fracture. However, the femoral fracture rate was higher in our twoincision group. We hypothesized that this was because the surgical field could not be fully visualized during stem implantation with the two-incision approach; this technique has an inherently high risk of complications that is difficult to minimize, even by surgeons with advanced proficiency in the procedure $[36,37]$.

There was a $5.3 \%$ revision rate for the stem in the twoincision group, including 2 periprosthetic fractures and 2 with aseptic loosening of the stem. With stem revision as the endpoint, survivorship in both groups for any reason was $97.3 \%$, and for aseptic loosening, it was $98.6 \%$ at 10 years. This is compatible with reports [38-41] on the durability of conventionally performed cementless THAs in cohorts with ONFH.

This study has some limitations. First, this was a retrospective study, and the cases were not randomized. However, all of the surgeries were done by the same surgeon using the same protocol and implants, which decreases the effects of some confounding factors. Second, the case number was modest: 75 hips in each group. One study [19], using the HHS scoring system to calculate a sample size with a power of $90 \%$ and a significance of 0.05 to detect a difference of 10 points in the HHS score (estimated SD of 15), found that 48 hips were required per group. Although our sample size was more than adequate to detect a difference in HHS, this study may still be underpowered to demonstrate significant differences. Finally, only clinical and radiological assessments were done. Functional analyses (gait and muscle strength) and other kinematic studies that might have explored more of the risks and benefits of different surgical approaches $[8,42]$ were not done.

In conclusion, both the two-incision and modified WJ approach provided satisfactory clinical results and survival rates at a mean follow-up of 10.8 years. However, there were high incidences of lateral femoral cutaneous nerve injury and periprosthetic femoral fracture when using the two-incision technique. We now routinely use the modified WJ approach for THAs in patients with ONFH. A prospective, randomized, large-scale cohort study is still warranted to provide evidence-based recommendations for patients with ONFH.

\section{Conflicts of Interest}

All authors state that they have no conflicts of interest. 


\section{Acknowledgments}

The authors wish to thank Miss Yu-Shuan Lin and Yu-yu Lin for their assistance in data collection and statistical analyses.

\section{References}

[1] M. S. Lee, P.-H. Hsieh, C.-H. Shih, and C.-J. Wang, "Nontraumatic osteonecrosis of the femoral head-from clinical to bench," Chang Gung Medical Journal, vol. 33, no. 4, pp. 351-360, 2010.

[2] P. J. Duwelius, "Two-incision minimally invasive total hip arthroplasty: techniques and results to date," Instructional Course Lectures, vol. 55, pp. 215-222, 2006.

[3] D. Chen and R. A. Berger, "Outpatient minimally invasive total hip arthroplasty via a modified Watson-Jones approach: technique and results," Instructional Course Lectures, vol. 62, pp. 229-236, 2013.

[4] K. C. Foucher, M. A. Wimmer, K. C. Moisio et al., "Time course and extent of functional recovery during the first postoperative year after minimally invasive total hip arthroplasty with two different surgical approaches-a randomized controlled trial," Journal of Biomechanics, vol. 44, no. 3, pp. 372-378, 2011.

[5] S. J. Wall and S. C. Mears, "Analysis of published evidence on minimally invasive total hip arthroplasty," The Journal of Arthroplasty, vol. 23, no. 7, article 55, 2008.

[6] P. J. Duwelius and L. D. Dorr, "Minimally invasive total hip arthroplasty: an overview of the results," Instructional Course Lectures, vol. 57, pp. 215-222, 2008.

[7] M. S. Lee, "Minimally invasive total hip arthroplasty," Formosan Journal of Musculoskeletal Disorders, vol. 2, no. 2, pp. 41-44, 2011.

[8] C.-C. Hu, J.-S. Chern, P.-H. Hsieh, C.-H. Shih, S. W. N. Ueng, and M. S. Lee, "Two-incision versus modified Watson-Jones total hip arthroplasty in the same patients-a prospective study of clinical outcomes and patient preferences," Chang Gung Medical Journal, vol. 35, no. 1, pp. 54-61, 2012.

[9] R. A. Berger, "Total hip arthroplasty using the minimally invasive two-incision approach," Clinical Orthopaedics and Related Research, no. 417, pp. 232-241, 2003.

[10] K. C. Bertin and H. Röttinger, "Anterolateral mini-incision hip replacement surgery: a modified Watson-Jones approach," Clinical Orthopaedics and Related Research, no. 429, pp. 248255, 2004.

[11] S. Amman, A. Cizik, S. S. Leopold, and P. A. Manner, "Twoincision minimally invasive vs standard total hip arthroplasty: comparison of component position and hospital costs," Journal of Arthroplasty, vol. 27, no. 8, pp. 1569-1574, 2012.

[12] J. D. Crowther and P. F. Lachiewicz, "Survival and polyethylene wear of porous-coated acetabular components in patients less than fifty years old: results at nine to fourteen years," Journal of Bone and Joint Surgery. A, vol. 84, no. 5, article 729, 2002.

[13] M. A. Mont, L. C. Jones, and D. S. Hungerford, "Nontraumatic osteonecrosis of the femoral head: ten years later," The Journal of Bone \& Joint Surgery-American Volume, vol. 88, no. 5, p. 1117, 2006.

[14] C. J. Della Valle, E. Dittle, M. Moric, S. M. Sporer, and A. Buvanendran, "A prospective randomized trial of mini-incision posterior and two-incision total hip arthroplasty," Clinical Orthopaedics and Related Research, vol. 468, no. 12, pp. 3348-3354, 2010.
[15] A. J. Krych, M. W. Pagnano, K. C. Wood, R. M. Meneghini, and K. Kaufmann, "No benefit of the two-incision THA over miniposterior THA: A Pilot Study of Strength and Gait," Clinical Orthopaedics and Related Research, vol. 468, no. 2, pp. 565-570, 2010.

[16] A. J. Krych, M. W. Pagnano, K. Coleman Wood, R. M. Meneghini, and K. Kaufman, "No strength or gait benefit of two-incision THA: a brief followup at 1 year," Clinical Orthopaedics and Related Research, vol. 469, no. 4, pp. 1110-1118, 2011.

[17] N. V. Greidanus, S. Chihab, D. S. Garbuz et al., "Outcomes of minimally invasive anterolateral THA are not superior to those of minimally invasive direct lateral and posterolateral THA," Clinical Orthopaedics and Related Research, vol. 471, no. 2, pp. 463-471, 2013.

[18] S. C. Graves, B. M. Dropkin, B. J. Keeney, J. D. Lurie, and I. M. Tomek, "Does surgical approach affect patient-reported function after primary THA?" Clinical Orthopaedics and Related Research, vol. 474, no. 4, pp. 971-981, 2016.

[19] J. Achten, N. R. Parsons, R. P. Edlin et al., "A randomised controlled trial of total hip arthroplasty versus resurfacing arthroplasty in the treatment of young patients with arthritis of the hip joint," BMC Musculoskeletal Disorders, vol. 11, pp. 8-13, 2010.

[20] C. A. Engh, P. Massin, and K. E. Suthers, "Roentgenographic assessment of the biologic fixation of porous-surfaced femoral components," Clinical Orthopaedics and Related Research, no. 257, pp. 107-128, 1990.

[21] R. C. Johnston, R. H. Fitzgerald Jr., W. H. Harris et al., "Clinical and radiographic evaluation of total hip replacement. A standard system of terminology for reporting results," The Journal of Bone and Joint Surgery, vol. 72, no. 2, pp. 161-168, 1990.

[22] Y.-H. Kim and V. E. M. Kim, "Uncemented porous-coated anatomic total hip replacement. Results at six years in a consecutive series," Journal of Bone and Joint Surgery. Series B, vol. 75, no. 1, pp. 6-13, 1993.

[23] B. Konigsberg, R. Hess, C. Hartman, L. Smith, and K. L. Garvin, "Inter- and intraobserver reliability of two-dimensional CT scan for total knee arthroplasty component malrotation," Clinical Orthopaedics and Related Research, vol. 472, no. 1, pp. 212-217, 2014.

[24] W. H. Harris, "Traumatic arthritis of the hip after dislocation and acetabular fractures: treatment by mold arthroplasty. An end-result study using a new method of result evaluation," The Journal of Bone \& Joint Surgery-American Volume, vol. 51, no. 4, pp. 737-755, 1969.

[25] N. Bellamy, W. W. Buchanan, C. H. Goldsmith, J. Campbell, and L. W. Stitt, "Validation study of WOMAC: a health status instrument for measuring clinically important patient relevant outcomes to antirheumatic drug therapy in patients with osteoarthritis of the hip or knee," Journal of Rheumatology, vol. 15, no. 12, pp. 1833-1840, 1988.

[26] B. S. Bal, D. Haltom, T. Aleto, and M. Barrett, "Early complications of primary total hip replacement performed with a two-incision minimally invasive technique," Journal of Bone and Joint Surgery. Series A, vol. 87, no. 11, pp. 2432-2438, 2005.

[27] B. A. Jewett and D. K. Collis, "High complication rate with anterior total hip arthroplasties on a fracture table," Clinical Orthopaedics and Related Research, vol. 469, no. 2, pp. 503-507, 2011.

[28] T. R. Yoon, K. S. Park, E. K. Song, J. K. Seon, and H. Y. Seo, "New two-incision minimally invasive total hip arthroplasty: comparison with the one-incision method," Journal of Orthopaedic Science, vol. 14, no. 2, pp. 155-160, 2009. 
[29] T. K. Fehring and J. B. Mason, "Catastrophic complications of minimally invasive hip surgery. A series of three cases," The Journal of Bone \& Joint Surgery-American Volume, vol. 87, no. 4, pp. 711-714, 2005.

[30] D. J. Berry, R. A. Berger, J. J. Callaghan et al., "Minimally invasive total hip arthroplasty. Development, early results, and a critical analysis. Presented at the Annual Meeting of the American Orthopaedic Association, Charleston, South Carolina, USA, June 14, 2003," The Journal of Bone and Joint Surgery. American Volume, vol. 85, no. 11, pp. 2235-2246, 2003.

[31] P. J. Duwelius, R. L. Burkhart, J. O. Hayhurst, H. Moller, and J. B. V. Butler, "Comparison of the 2-incision and mini-incision posterior total hip arthroplasty technique. A Retrospective Match-Pair Controlled Study," Journal of Arthroplasty, vol. 22, no. 1, pp. 48-56, 2007.

[32] Y.-H. Gao, S.-Q. Li, Y.-F. Wang, C. Yang, J.-G. Liu, and X. Qi, "Arthroplasty in patients with extensive femoral head avascular necrosis: cementless arthroplasty in extensive femoral head necrosis," International Orthopaedics, vol. 39, no. 8, pp. 15071511, 2015.

[33] J. D. Calder, M. F. Pearse, and P. A. Revell, "The extent of osteocyte death in the proximal femur of patients with osteonecrosis of the femoral head," Journal of Bone and Joint SurgerySeries B, vol. 83, no. 3, pp. 419-422, 2001.

[34] Y.-H. Kim and J.-S. Kim, "Histologic analysis of acetabular and proximal femoral bone in patients with osteonecrosis of the femoral head," Journal of Bone and Joint Surgery - Series A, vol. 86, no. 11, pp. 2471-2474, 2004.

[35] M. Ollivier, S. Parratte, T. Lecorroller, A. Reggiori, P. Champsaur, and J.-N. Argenson, "Anatomy of the proximal femur at the time of total hip arthroplasty is a matter of morphotype and etiology but not gender," Surgical and Radiologic Anatomy, vol. 37, no. 4, pp. 377-384, 2015.

[36] N. V. Greidanus, S. Chihab, D. S. Garbuz et al., "Outcomes of minimally invasive anterolateral THA are not superior to those of minimally invasive direct lateral and posterolateral THA hip," Clinical Orthopaedics and Related Research, vol. 471, no. 2, pp. 463-471, 2013.

[37] M. W. Pagnano, J. Leone, D. G. Lewallen, and A. D. Hanssen, "Two-incision THA had modest outcomes and some substantial complications," Clinical Orthopaedics and Related Research, no. 441, pp. 86-90, 2005.

[38] D. W. Chen, C.-C. Hu, Y.-H. Chang, W.-E. Yang, and M. S. Lee, "Comparison of clinical outcome in primary total hip arthroplasty by conventional anterolateral transgluteal or 2incision approach," Journal of Arthroplasty, vol. 24, no. 4, pp. 528-532, 2009.

[39] S.-M. Kim, S.-J. Lim, Y.-W. Moon, Y.-T. Kim, K.-R. Ko, and Y.S. Park, "Cementless modular total hip arthroplasty in patients younger than fifty with femoral head osteonecrosis: minimum fifteen-year follow-up," Journal of Arthroplasty, vol. 28, no. 3, pp. 504-509, 2013.

[40] S.-I. Han, J.-H. Lee, J. W. Kim, C. W. Oh, and S.-Y. Kim, "Longterm durability of the CLS femoral prosthesis in patients with osteonecrosis of the femoral head," Journal of Arthroplasty, vol. 28, no. 5, pp. 828-831, 2013.

[41] B.-W. Min, K.-J. Lee, K.-S. Song, K.-C. Bae, and C.-H. Cho, "Highly cross-linked polyethylene in total hip arthroplasty for osteonecrosis of the femoral head: a minimum 5-year follow-up study," Journal of Arthroplasty, vol. 28, no. 3, pp. 526-530, 2013.

[42] C.-J. Chang, N.-L. Lin, M. S. Lee, and J.-S. Chern, "Recovery of posture stability at different foot placements in patients who underwent minimally invasive total hip arthroplasty: a oneyear follow-up study," BioMed Research International, vol. 2015, Article ID 463792, 10 pages, 2015. 


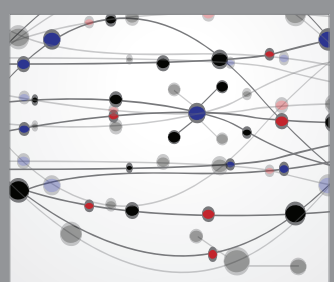

The Scientific World Journal
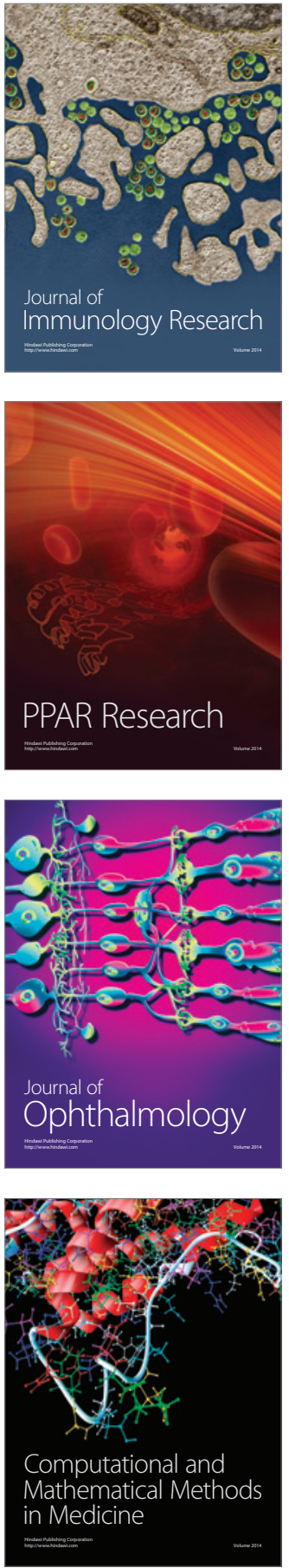

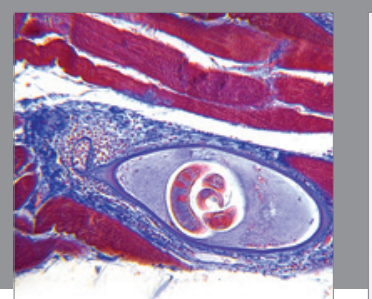

Gastroenterology Research and Practice
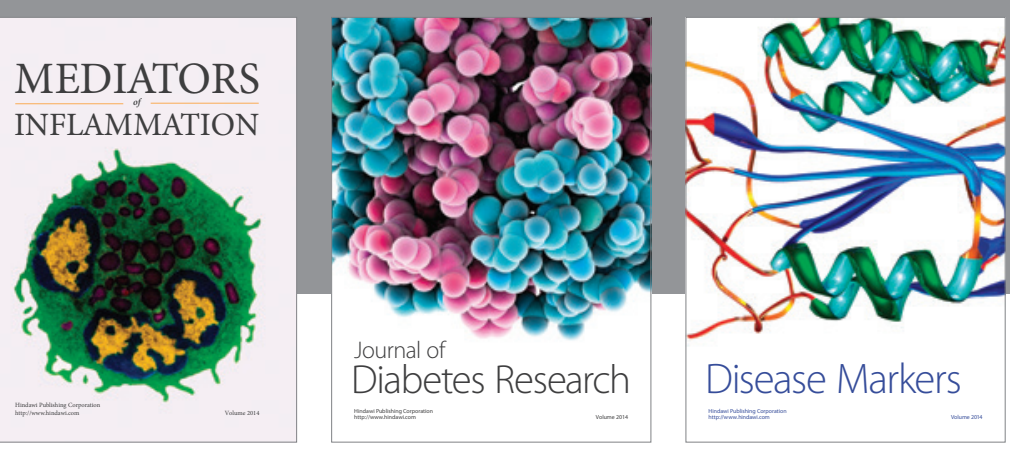

Disease Markers

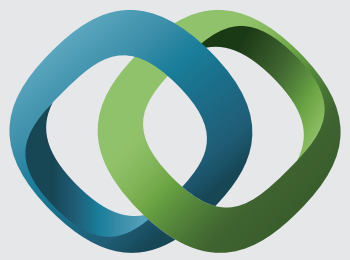

\section{Hindawi}

Submit your manuscripts at

https://www.hindawi.com
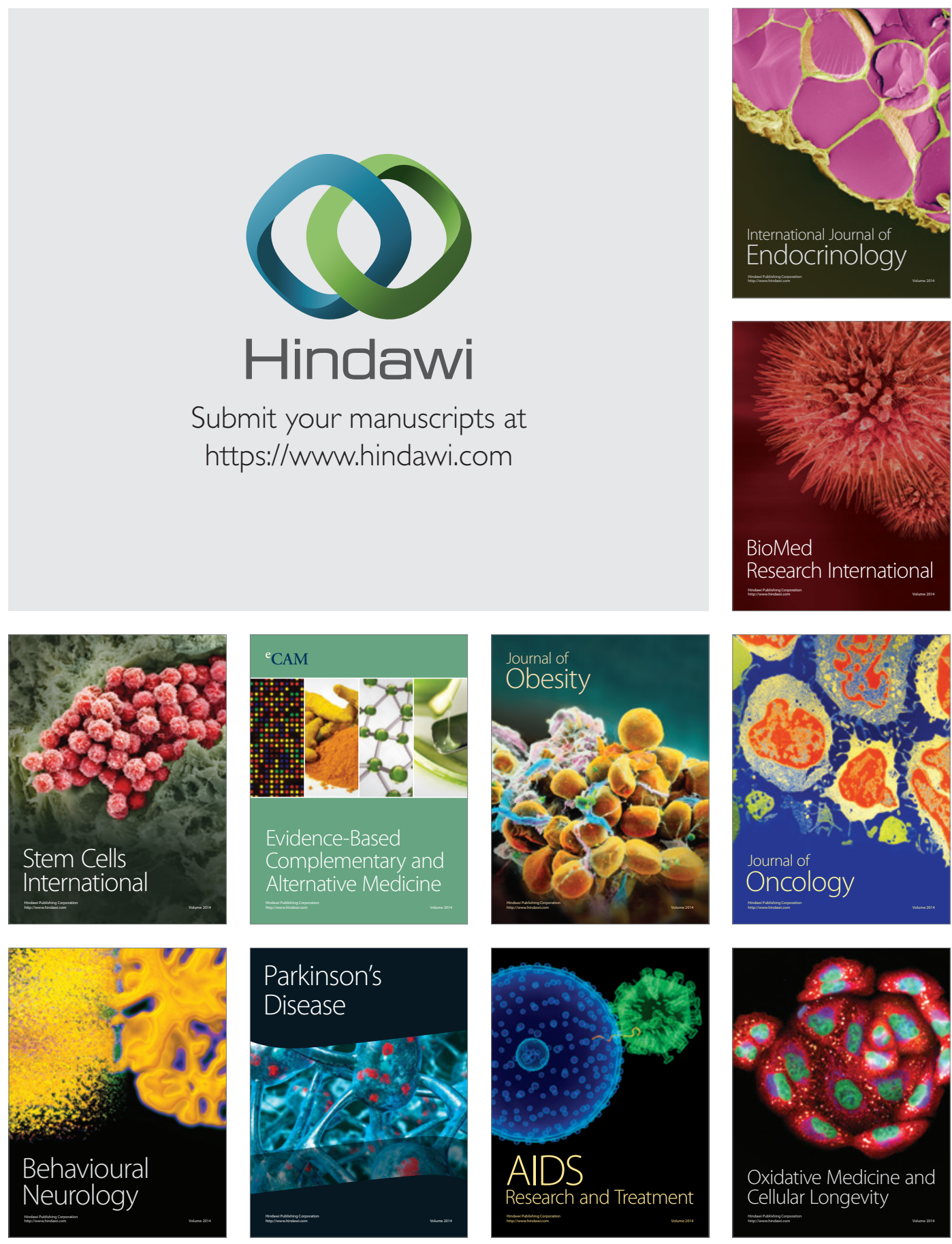